\title{
Nutzen von Azithromycin bei Bronchialveränderungen
}

\author{
Die Rate an Exazerbationen bei Bronchiektasen wird mit \\ 1,5-6,5 pro Patient und Jahr angegeben. Für deren Prävention \\ und Behandlung stehen nur wenige evidenzbasierte Therapien \\ zur Verfügung. C. Wong et al. haben nun den Nutzen des \\ Makrolidantibiotikums Azithromycin bei Patienten mit nicht \\ mukoviszidosebedingten Bronchiektasen untersucht. \\ Lancet 2012; 380: 660-667
}

An der randomisierten, doppelblinden EMBRACE-Studie (Effectiveness of Macrolides in Patients with Bronchiectasis using Azithromycin to control Exacerbations) nahmen Patienten teil, die folgende Kriterien erfüllten: Alter $\geq 18$ Jahre, mindestens eine behandlungsbedürftige Exazerbation im vergangenen Jahr und eine CT-bestätigte Brochiektase-Diagnose. Die Patienten erhielten entweder $500 \mathrm{mg}$ Azithromycin oder Placebo an 3 Tagen in der Woche über insgesamt 6 Monate. Es folgte ein Followup von weiteren 6 Monaten. Untersuchungen fanden nach 4, 13, 26, 39 und 52 Wochen statt; außerdem wurden die Patienten zwischen diesen Terminen telefonisch befragt. Neben der Spirometrie beurteilten die Mediziner auch Daten aus dem St. George's Respiratory Questionnaire (SGRQ), dem 6-Minuten-Gehtests, Messungen des C-reaktiven Proteins (CRP) und zu Nebenwirkungen. Die Endpunkte dieser Studie waren ereignisbasierte Exazerbationen innerhalb der ersten 6 Monate, FEV $_{1}$ und SGRQ-Score am Therapieende.

\section{Weniger Exazerbationen \\ $\nabla$}

Insgesamt konnten 71 Teilnehmer in die Azithromycin- und 70 in die Placebogruppe randomisiert zugeordnet werden. Die Adhärenz betrug im Verum- 97,9\% und im Placeboarm 98,3\%. Während es unter dem Antibiotikum nach 6 Monaten zu 42 Exazerbationen kam, traten unter Placebo 103 Ereignisse auf. Daraus ergaben sich ereignisbasierte Exazerbationsraten von 0,59 (Azithromycin) und 1,57 (Placebo) mit einer relativen Reduktion von $62 \%$ ( $p<0,0001)$. Die entsprechenden Ergebnisse nach 12 Monaten waren: 109 Exazerbationen (Azithromycin), 178 Exazerbationen (Placebo), Exazerbationsraten 1,58 bzw. 2,73, relative Reduktion zugunsten von Azithromycin 42\% (p<0,0001). Da 31\% der Verum- und 66\% der Placebopatienten in 6 Monaten mindestens 1 ereignisbasierte Exazerbation erlitten, erreichte die Risikoreduktion 52\%( $\mathrm{p}<0,0001)$; nach $12 \mathrm{Mo-}$ nate waren es $25 \%(0,005)$. Die Zeit, in der es bei mindestens $25 \%$ der Patienten nach 6 Monaten zur ersten Exazerbation kam, lag im Verum- bei 104, im Placeboarm bei 21 Tagen $(\mathrm{p}<0,0001)$. Nach 12 Monaten betrugen die Zeiträume 239 vs. 85 Tage $(\mathrm{p}<0,0001)$.

Weder die symptombasierten Exazerbationen noch die $\mathrm{FEV}_{1}$-Veränderungen (Prä-, Post-Bronchodilatation) ergaben deutliche Unterschiede zwischen den Studienarmen. Gleiches galt auch für den SGRQ-Gesamtscore. Die CRP-Konzentrationen, die nur nach 6 Monaten gemessen wurden, gingen im Vergleich zu den Ausgangswerten unter Azithromycin zurück und stiegen in der Placebogruppe an (-13,8 vs. $40,1 \%$, $\mathrm{p}=0,006$ ). Die Verumtherapie wurde allgemein gut vertragen und verursachte überwiegend leichte gastrointestinale Nebenwirkungen.

\section{Fazit}

Patienten mit nicht mukoviszidosebedingten Bronchiektasen hatten in der Studie mit der Azithromycin-Therapie weniger Exazerbationen. Bis zur ersten Verschlimmerung verging eine längere Zeit. Dieser Nutzen hielt 6 Monate nach Behandlungsende an. Die Autoren empfehlen eine sorgfältige Patientenauswahl und weisen auf das Risiko von Macrolidresistenzen hin.

\section{Matthias Manych, Berlin}

\section{Rauchen \\ Rauchausstieg auch im Alter effektiv}

Raucher steigern mit jeder Zigarette ihr Herzinfarkt- und Schlaganfall-Risiko. Doch auch umgekehrt gilt: Selbst wer erst im fortgeschrittenen Alter mit dem Rauchen aufhört, senkt sein Risiko bereits innerhalb kürzester Zeit nach dem Ausstieg erheblich. Das fanden Forscher des Deutschen Krebsforschungszentrums (DKFZ) jetzt anhand einer Studie an Einwohnern aus dem Saarland heraus.

Für ihre Studie analysierten Prof. Hermann Brenner und Kollegen die Daten von 8807 Personen im Alter von 50-74 Jahren. „Wir konnten zeigen, dass Raucher ein mehr als doppelt so hohes Risiko für Herz-Kreislauf-Erkrankungen haben wie Nichtraucher. Ehemalige Raucher sind dagegen fast genauso selten betroffen wie Menschen gleichen Alters, die nie geraucht haben“, sagt Brenner. „Außerdem erkranken Raucher deutlich früher als Personen, die nicht oder nicht mehr rauchen." So hat beispielsweise ein 60-jähriger Raucher das Herzinfarkt-Risiko eines 79-jährigen Nichtrauchers und das Schlaganfall-Risiko eines 69-jährigen Nichtrauchers. Dabei wirken sich die Tabakdosis und die Dauer des Konsums auf das Erkrankungsrisiko aus: Je mehr Zigaretten pro Tag über einen längeren Zeitraum geraucht werden, desto höher ist das Risiko.

Der positive Effekt eines Rauchausstiegs macht sich bei den Studienteilnehmern bereits nach kurzer Zeit bemerkbar. „Verglichen mit Personen, die weiterhin rauchen, ist das Risiko für einen Herzinfarkt und für einen Schlaganfall bereits während der ersten 5 Jahre nach der letzten Zigarette mehr als $40 \%$ niedriger“, betont Carolin Gellert, die Erstautorin der Studie, die Anfang 2013 im „European Journal of Epidemiology“ veröffentlicht wurde. Die Ergebnisse legen nahe, dass Programme zur Tabakentwöhnung, die sich bislang auf jüngere Teilnehmer konzentrieren, auf ältere Personen ausgeweitet werden sollten.

Nach einer Mitteilung des DKFZ, Heidelberg 